\title{
Medical Images Fusion Based on Different Frequency band Selection Schemes
}

\author{
Shuangfang $\mathrm{Yu}{ }^{1}$, Ying Wang ${ }^{2}$ and Wenbing Fan ${ }^{3}$ \\ School of Information Engineering, Zhengzhou University, Henan, 450000, China \\ 11159729983@qq.com ,2 ieywang@zzu.edu.cn,3iewbfan@zzu.edu.cn
}

Keywords:Image fusion, PLIP model, curvelet transform, sub-band selection scheme

\begin{abstract}
A novel curvelet-based sub-band selection approach for medical image fusion is presented in this paper using the Parameterized Logarithmic Image Processing (PLIP) model. Both MRA and MRI image have different features, in this paper we use the different frequency band selection fusion rules yield novel fusion schemes for combining the coefficients, and fused MRA and MRI images to form a single image with as much information as possible. The experiments show that the proposed method is effective and can get satisfactory fusion results.
\end{abstract}

\section{Introduction}

In the recent years, the study of medical image fusion attracts much attention including diagnosis, research, and treatment, with the rapid development in high-technology and modern instrumentations. Image fusion is the combination of multimodality source images which vary in resolution, instrument modality, or image capture technique into a single composite representation [1]. Multimodality medical images mainly include the following images, such as X-ray, computed tomography (CT), magnetic resonance imaging (MRI), magnetic resonance angiography(MRA) and so on [2]. With more available multimodality medical images in clinical applications, the idea of combining images from different modalities becomes very important and medical image fusion has merged as a new and promising research field. The main objective of medical imaging is to obtain a high resolution image with as much details as possible for the sake of diagnosis. MR and the CT techniques are medical imaging techniques. Both techniques give special sophisticated characteristics of the organ to be imaged. So, it is expected that the fusion of the MR and the CT images of the same organ would result in an integrated image of much more details. Due to this question, the application of the curvelet transform use the different frequency band selection fusion rules for image fusion is introduced in this paper.

\section{Curvelet Transform}

The curvelet transform has evolved as a tool for the representation of curved shapes in graphical applications. Then, it was extended to the fields of edge detection and image denoising.The first generation curvelet transform is more complex involves a series of steps. Due to its complexity, the second generation curvelet is much preferred. The detailed fusion steps based on curvelet transform can be summarized below.

\section{Wrapping Algorithm}

(1) Perform FFT on the original image.

(2) Divide FFT into collection of tiles .

(3) For each tile apply

a. Translate tile to the origin.

b. Wrap parallelogram shaped support of tile around the rectangle with center .

c. Take inverse FFT of wrapped one

d. Add curvelet array to collection of curvelet coefficients.

Inverse Wrapping Algorithm

(1) For each curvelet coefficient array 

a. Take FFT of the array.
b. Unwrap rectangular support to original orientation shape.
c. Translate it back to the original position
d. Store the translated array
(2) Add all the translated curvelet arrays
(3) Take inverse FFT to reconstruct the image.

\section{Image fusion using curvelet transform of PLIP Model}

Since the main objective of image fusion is to fuse the multimodal medical images, the characteristics of the images should also be considered. For the fusion example of MRA and MRI images.

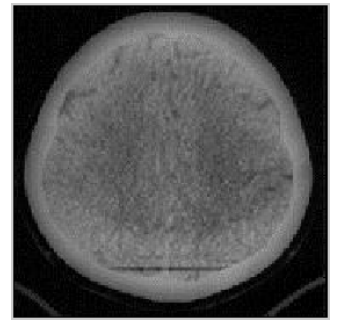

Fig.1 Curvelet transform of MRI Image
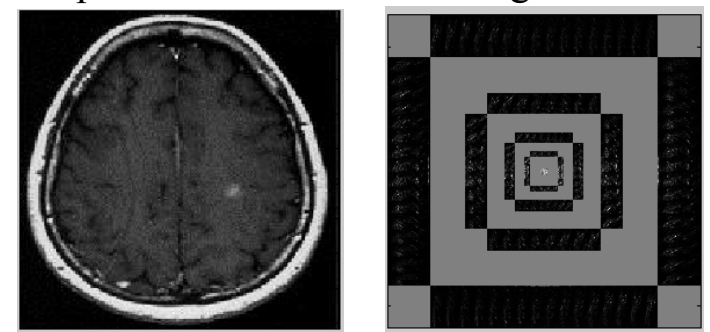

Fig.2 Curvelet transform of MRA Image

\section{Weighted Average method in low frequency sub-band}

The approximation coefficients at the highest level of decomposition are most commonly fused via uniform averaging because of its mean intensity value of the source images and minimal loss of salient features [6]. While the weighted average method proposed in [12] remains one of the most effective after more than ten years since its inception. The method is based on a local-Gaussian assumption for wavelet sub-bands. In this section, the weighted average method can be reformulated and modified in order to cope with more appropriate statistical assumptions like the generalized Gaussian based on [12] and [13]. We also consider only two original images, $A$ and $B$, and their fused image $Y$. And the multi scale decompositions of the original and fused images are denoted by $C_{A}$, $C_{B}$ and $C_{Y}$.

$$
\begin{gathered}
\mu(p)=\frac{1}{N^{2}} \sum_{(i, j) \in W_{N}} C(m+i, n+j, k, l) \\
\Upsilon(\mu(p))=\left(\frac{1}{\mu(p)}\right)^{\alpha} \\
\varpi(p)=\frac{1}{N^{2}} \sum_{(i, j) \in W_{N}} \Upsilon(\mu(p)) \cdot \frac{|C(m+i, n+j, k, l)-\mu(p)|}{\mu(p)}
\end{gathered}
$$

where $W_{N}$ is a $N \times N$ block, $\varpi(p)$ is the weighting factor, $\alpha$ is a constant by perceptual experiment, and it has a peak, when around peak values can achieve very good effect, too big or too small can't achieve the ideal effect. After calculating the visibility of all the coefficients in the low-frequency band, the approximation coefficients for the fused image $Y$ at the highest level of decomposition use weighted averaging by

$$
C_{Y}(p)=\frac{\varpi_{A}(p) \cdot C_{A}(p)+\varpi_{B}(p) \cdot C_{B}(p)}{\varpi_{A}(p)+\varpi_{B}(p)}
$$

\section{Fusion Scheme in high frequency sub-bands}

For the high-frequency bands, it is generally believed that the details of an image are mainly included in the high-frequency of the image. Therefore, it is important to select a suitable image fusion algorithm that produces maximizing amount of information in fused image. As we know a pixel in an image must have some relations with its neighboring pixels, which means that a decomposed wavelet coefficient will also have relations with its neighboring coefficients. So we propose a scheme by calculating the neighborhood variance of high frequency sub-bands to select the high-frequency coefficients using PLIP-curvelet model. The procedure can be formulated as follows.

For each pixel of the $l$ high frequency sub-band at $k$ level of decomposition, the neighborhood 
variance of the two input image detail coefficients $C_{A}(p), C_{B}(p)$ and their covariance are determined by

$$
\begin{aligned}
\sigma_{A}(p) & =\frac{1}{N^{2}} \sum_{(i, j) \in W_{N}}\left(C_{A}(m+i, n+j, k, l)-\mu_{A}(p)\right)^{2} \\
\sigma_{B}(p) & =\frac{1}{N^{2}} \sum_{(i, j) \in W_{N}}\left(C_{B}(m+i, n+j, k, l)-\mu_{B}(p)\right)^{2} \\
\mu_{A}(p) & =\frac{1}{N^{2}} \sum_{(i, j) \in W_{N}} C_{A}(m+i, n+j, k, l) \\
\mu_{B}(p) & =\frac{1}{N^{2}} \sum_{(i, j) \in W_{N}} C_{B}(m+i, n+j, k, l) \\
\sigma_{A B}(p)= & \frac{1}{N^{2}} \sum_{(i, j)) \in W_{N}}\left(C_{A}(m+i, n+j, k, l) \cdot C_{B}(m+i, n+j, k, l)-\mu_{A B}(p)\right)^{2} \\
\mu_{A B}(p) & =\frac{1}{N^{2}} \sum_{(i, j) \in W_{N}} C_{A}(m+i, n+j, k, l) \cdot C_{B}(m+i, n+j, k, l)
\end{aligned}
$$

The local matching coefficient measure of each sub-band between source images is given as

$$
M_{A B}(p)=\frac{2 \sigma_{A B}(p)}{\sigma_{A}^{2}(p)+\sigma_{B}^{2}(p)}
$$

Comparing the matching measure to a threshold $T$ determines if detail coefficients are to be combined by simple selection or by weighted averaging.

$$
\delta(p)= \begin{cases}1-\frac{M_{A B}(p)-T}{2(1-T)}, & \sigma_{A}(p)>\sigma_{B}(p), \\ \frac{M_{A B}(p)-T}{2(1-T)}, & \sigma_{A}(p)<\sigma_{B}(p), \\ 1 & M_{A B}(p)>T \\ & \sigma_{A}(p)>\sigma_{B}(p), \\ & M_{A B}(p)>T \\ 0 & \sigma_{A}(p)<\sigma_{B}(p), \\ & M_{A B}(p)>T\end{cases}
$$

where $\delta(p)$ indicts the factor of multiplicative weight averaging. The fused coefficients are calculated using the following formula.

$$
C_{Y}(p)=\delta(p) \cdot C_{A}(p)+(1-\delta(p)) \cdot C_{B}(p)
$$

This method cannot guarantee the homogeneity in the resultant fused image, especially for the high frequency sub-bands. In this paper we apply a window-based verification to the fused high frequency coefficients.

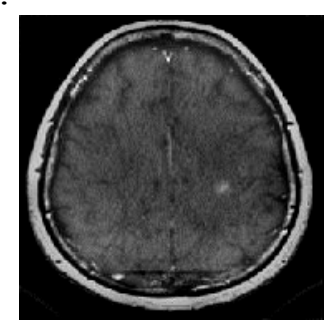

(a)

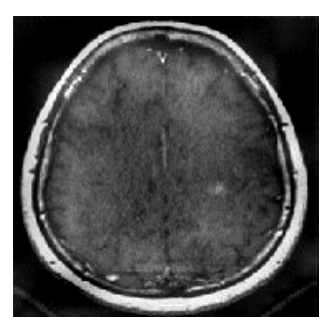

(b)

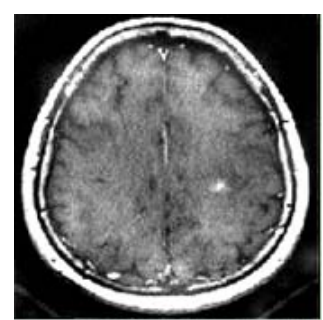

(c)

Fig.3 Different fusion results of the different weighting factor.(a) when $\alpha=0.2$;(b) when $\alpha=-3.0$;(c) when $\alpha=-1.3$.

\section{Conclusions}

In this paper, we present a novel curvelet-based sub-band selection approach for medical image fusion is presented in this paper using the Parameterized Logarithmic Image Processing (PLIP) model. Firstly, from the comparison of experimental results, it is seen that the curvelet decomposition using the PLIP model provides the best balance between extracting the soft tissues information, scarf, pathological edges, and their features in the image. Secondly, the fused image is reconstructed by all using composite coefficients and inverse isomorphic transform. The experimental results showed that 
the proposed fusion method out perform some existing fusion methods by both qualitative and quantitative means and it can get satisfactory fusion results.

\section{Acknowledgment}

This work was supported by our supervisor, Professor Wenbing Fan, School of Information Engineering, and by the Library of Zhenzhou University. The authors also thank the anonymous referees for their valuable suggestions.

\section{References}

[1] G.Piella, "A general framework for multiresolution image fusion: from pixels to regions," Information Fusion, vol. 4, no.4, pp. 259-280, 2003.

[2] F.Maes, D.Vandermeulen, and P.Suetens, "Medical image registration using mutual information," Proceedings of the IEEE, vol.91, no.10, pp.1699-1721, 2003.

[3] Z.Zhang and R.S.Blum, "A categorization of multi-scale decomposition-based image fusion schemes with a performance study for a digital camera application", Proceedings of the IEEE, vol. 87, no. 8, pp. 1315-1326, 1999.

[4] Y.Yang, D.S.Park, S.Huang, and N.Rao, "Fusion of CT and MR images using an improved wavelet based method", Journal of X-Ray Science and Technology, vol.18, no.10, pp.157-170, 2010.

[5] S.C.Nercessian, K.A.Panetta, and S.S.Agaian, "Multi-resolution decomposition schemes using the parameterized logarithmic image processing model with application to image fusion", EURASIP Journal on Advances in Signal Processing, Vol.2011, Article ID 515084, 17 pages, 2011.

[6] M. Jourlin and J. C. Pinoli, "Logarithmic image processing: the mathematical and physical framework for the representation and processing of transmitted images", Advances in Imaging and Electron Physics, vol. 115, pp. 126-196, 2001.

[7] E. J . Candes , L . Demanet, D. L . Donoho et al . Fast DiscreteCurvelet Transforms [ R ] . A p plied and Com p utationalM at hematics . California Instit ute of Technology , 2005. 1 43.

[8] F.Chenab, Z.Guanc, and X.K.Yang, "A novel remote sensing image fusion method based on independent component analysis", International Journal of Remote Sensing, vol.32, no.10, pp.2745-2763, 2011.

[9] P.J.Burt and E.H.Adelson, "Lapacian pyramid as a compact image code," IEEE Transactions on Communications, vol. 31, no.4, pp.532-540,1983.

[10] H.Li, B.S.Manjunath, S.K.Mitra, "Multisensor image fusion using the wavelet transform", Graphical Models Image Process, vol.57, no.3, pp.235-245,1995.

[11] P.J. Burt and R.J. Kolczynski, "Enhanced image capture through fusion", in Proc.Fourth Int. Conf. on computer Vision, pp. 173-182, 1993.

[12] A. Cardinali and G.P. Nason, "A statistical multiscale approach to image segmentation and fusion", in Proceedings of the 8th International Conference on Information Fusion (Fusion 2005), Philadelphia, PA, USA, 25-29 July, 2005. 